\title{
Variable Stiffness Actuator for Soft Robotics Using Dielectric Elastomer and Low-Melting-Point Alloy
}

\author{
Jun Shintake ${ }^{1,2}$, Bryan Schubert ${ }^{1}$, Samuel Rosset ${ }^{2}$, Herbert Shea ${ }^{2}$ and Dario Floreano ${ }^{1}$
}

\begin{abstract}
A novel variable stiffness actuator composed of a dielectric elastomer actuator (DEA) and a low-melting-pointalloy (LMPA) embedded silicone substrate is demonstrated. The device which we call variable stiffness dielectric elastomer actuator (VSDEA) enables functional soft robots with a simplified structure, where the DEA generates a bending actuation and the LMPA provides controllable stiffness between soft and rigid states by Joule heating. The entire structure of VSDEA is made of soft silicones with an elastic modulus of less than $1 \mathrm{MPa}$ providing a high compliance when the LMPA is active. The device has the dimension of $40 \mathrm{~mm}$ length $\times 10 \mathrm{~mm}$ width $\times$ $1 \mathrm{~mm}$ thickness, with mass of $\sim 1 \mathrm{~g}$. We characterize VSDEA in terms of the actuation stroke angle, the blocked force, and the reaction force against a forced displacement. The results show the controllable actuation angle and the blocked force up to $23.7^{\circ}$ and $2.4 \mathrm{mN}$ in the soft state, and $0.6^{\circ}$ and $2.1 \mathrm{mN}$ in the rigid state. Compared to an actuator without the LMPA, VSDEA exhibits $\sim 90 \times$ higher rigidity. We develop a VSDEA gripper where the mass of active parts is $\sim 2 \mathrm{~g}$, which is able to successfully hold an object mass of $11 \mathrm{~g}$, exhibiting the high performance of the actuator.
\end{abstract}

\section{INTRODUCTION}

Soft robotics, an approach where robots are made from compliant materials, is a new trend that has demonstrated many potential applications, such as legged locomotion and grasping of fragile objects with a simple structure and control [1], [2], and biomimetic structures and locomotion [3]. Additionally, soft robots have a number of advantages over traditional robots, such as improved mechanical robustness [4].

Controllable stiffness can be an important function for soft robots to exert large forces to environments, and to withstand external loads while keeping their shape. This function has enabled multi-task movement [5], adapted terrestrial locomotion [6], handling of various objects [7], and improved dexterity in manipulation [8]. Toward further development of these types of functional soft robots, one approach is to use variable stiffness actuators in which the actuation and the controllable stiffness are provided by a single device, thus drastically simplifying the robotic structure. Technologies related to this purpose in the literature are based on jamming [6]-[8], electrorheological/magnetorheological fluids

This work was supported by the Swiss National Centre of Competence in Research (NCCR) Robotics, and the Swiss National Science Foundation grant 200020-153122.

${ }^{1}$ J. Shintake, B. Schubert and D. Floreano are with the Laboratory of Intelligent Systems (LIS), École Polytechnique Fédérale de Lausanne, Route Cantonale, Lausanne 1015, Switzerland. jun. shintake@epfl.ch

${ }^{2}$ J. Shintake, S. Rosset and H. Shea are with the Microsystems for Space Technologies Laboratory (LMTS), École Polytechnique Fédérale de Lausanne, Rue de la Maladière 71B, Neuchâtel 2000, Switzerland.

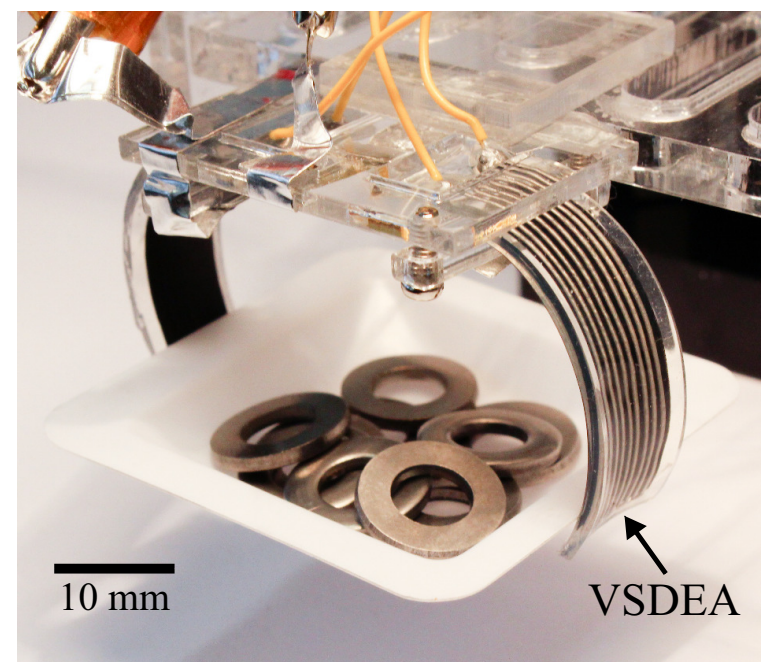

Fig. 1. Developed gripper, consisting of two VSDEAs, holding a plastic dish filled with metal washers of mass $11 \mathrm{~g}$ in its rigid state (mass of active parts is $\sim 2 \mathrm{~g}$ ). The operating procedure of the gripper can be seen on [28].

[9], [10], shape memory polymers [11], [12], and lowmelting-point alloys (LMPAs) [5]. However, these examples use external parts to provide air pressure, electromagnetic field, and temperature, respectively, which could lead to a complicated structure. Shape memory alloy could be a candidate as it is able to change its stiffness by Jouleheating directly, but it exhibits a small relative stiffness change $(\sim 2.3 \times)$ and a high absolute stiffness (13 GPa elastic modulus in martensite phase) [13].

In this paper, we demonstrate a novel variable stiffness actuator composed of a dielectric elastomer actuator (DEA) [14], [15] and a LMPA embedded in a silicone substrate. The device, which we call variable stiffness dielectric elastomer actuator (VSDEA), can change the stiffness by direct Joule-heating of the LMPA, and the DEA provides bending actuation. The entire structure is made with soft silicone with elastic modulus of less than $1 \mathrm{MPa}$, providing a high compliance. As an application to demonstrate the usefulness of the VSDEA, we also develop a gripper shown in Fig. 1 where the actuators act as fingers.

DEAs and the LMPA substrate are further explained in section II. The mechanism, the fabrication process, and the characterization results of the VSDEA, and a further explanation of the gripper are shown in section III, followed by the discussion and the conclusions in section IV.

\section{DEA AND LMPA SUBSTRATE}

DEAs consist of an elastomer membrane sandwiched between compliant electrodes. Applying a voltage to the elec- 
trodes leads to generation of opposing charges on both sides of the membrane which cause area expansion and thickness reduction as the actuation. DEAs are soft $(\sim 1 \mathrm{MPa})$, thin (from several tens of $\mu \mathrm{m}$ ) therefore lightweight, fast $(\mathrm{kHz}$ bandwidth [16]), and exhibit large actuation strokes (over $1000 \%$ of area strain [18]), and multi-functional capabilities: switching and self-sensing capability [15], [17]. On the other hand, DEAs usually generate low forces, and a high voltage (a few $\mathrm{kV}$ ) is necessary for the actuation. DEAs have been applied for robotic applications including legged robots [19], [20], a multi-segmented snake-like robot [21], grippers [22], [23], an airship propelled by fish-like movement [24], and a micro air vehicle [25].

We previously developed LMPA substrate which showed a relative stiffness change of $>25 \times$ (elastic modulus of $40 \mathrm{MPa}$ in the rigid state, and $1.5 \mathrm{MPa}$ in the soft state), and a fast transition from rigid to soft states $(<1$ s) at a low power $(<0.5 \mathrm{~W})$ [26]. The substrate is made of a LMPA channel encapsulated in a silicone matrix where the LMPA changes its phase between solid and liquid by direct Joule-heating. Therefore, varying degrees of stiffness of the structure, between fully rigid and completely soft, are achieved efficiently as a function of the input power. The transition speed, power consumption, and both the absolute and the relative stiffness depend on the geometry of the LMPA channel and the silicone matrix. The LMPA substrate can also act as a strain sensor by measuring its change in resistance due to changes in geometry.

The DEA and the LMPA substrate can be made with silicone, and this inherent material compatibility eases the fabrication process of the VSDEA and simplifies the structure.

\section{VARIABLE STIFFNESS ACtUATOR}

\section{A. Mechanism}

VSDEA consists of a pre-stretched DEA attached onto a LMPA substrate, as shown in Fig. 2(a). We explain the actuation mechanism using Fig. 2(b). Even though the LMPA substrate can vary the stiffness as a function of the input power, in the rest of this paper we consider only the two extreme cases for the LMPA: solid and liquid. When the LMPA is activated, Joule-heating changes the phase of the alloy from solid to liquid, resulting in a soft state. At this state, (i) the entire structure is bent with a radius of curvature that minimizes the total energy of the device (i.e. the strain energy of the DEA and the bending energy of the substrate). (ii) Applying a voltage to the DEA while keeping the LMPA soft leads to a bending actuation towards the flat state, due to the electrostatic energy of the DEA which shifts the total energy. The amount of the bending can be controlled by the voltage on the DEA. (iii) If the LMPA is deactivated but the DEA is kept active until the structure becomes solid, (iv) a rigid state for a desired bending (or flat) shape can be achieved. At this state, the device can sustain its rigid shape without any input power. From this state, other rigid shapes, e.g., (v), can be achieved by activating the LMPA through the state (i). Rigid states (iv) and (v) mean the device is able
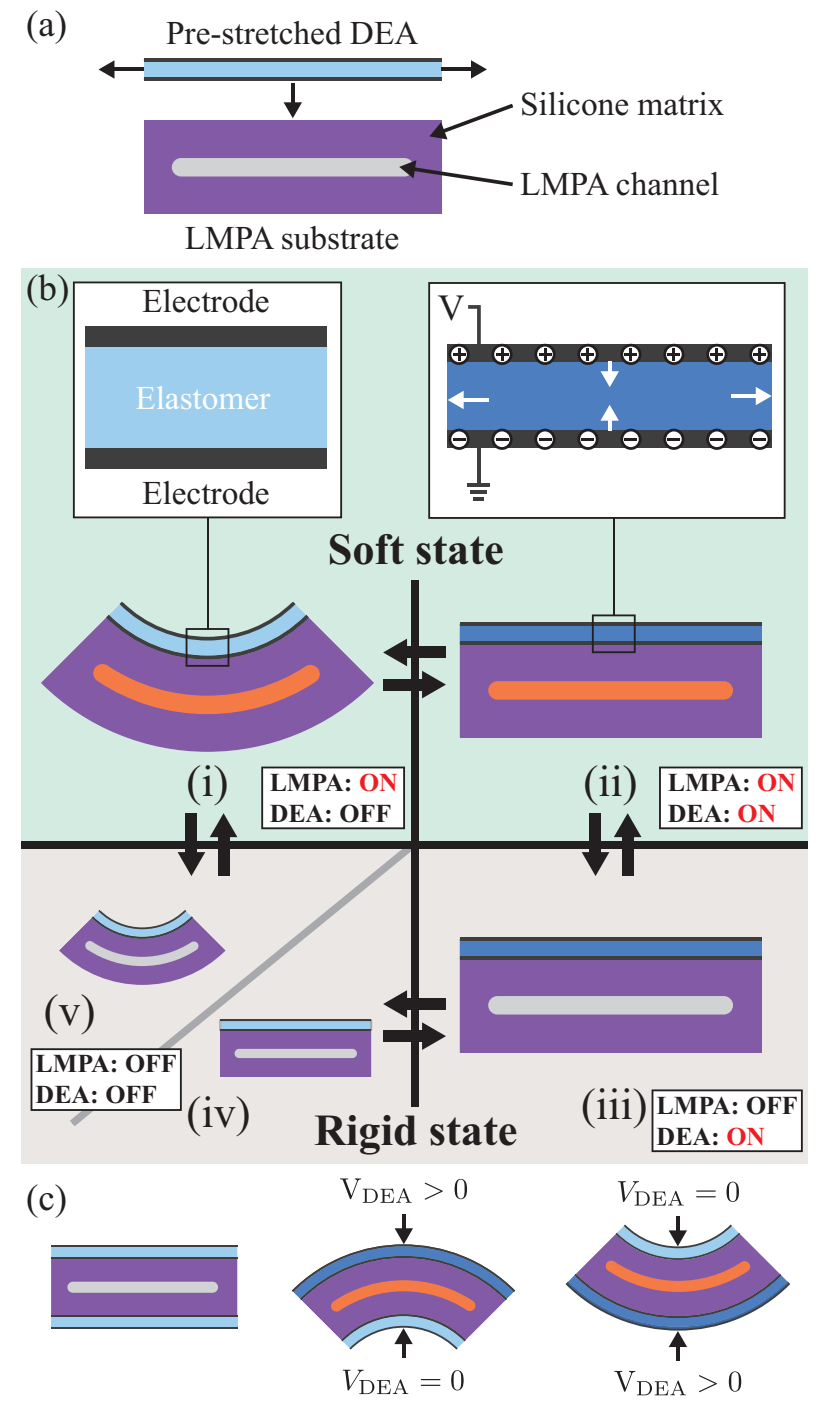

Fig. 2. Mechanism of VSDEA. (a) The actuator consists of a pre-stretched DEA attached onto a LMPA substrate. (b)(i)The activation of the LMPA makes the structure soft, resulting in a bending shape. (ii) At this state, applying a voltage to the DEA leads to a bending actuation toward the flat shape. (iii)(iv) Deactivation of the LMPA keeps a desired bending shape. (v) Re-activation of the LMPA through state (i) allows changing of the rigid shape. (c) Bidirectional actuation can be obtained by putting another DEA on the other side of the LMPA substrate.

to rigidify all the shapes that are achievable in the soft state. In addition, the actuation of VSDEA can be extended to bidirectional movement when another DEA is placed on the other side of the LMPA substrate, forming an antagonistic configuration as shown in Fig. 2(c). In this configuration, the DEAs start actuation from fully pre-stretched state, meaning that the actuation strain is beyond the pre-strain. Therefore, the performance of the actuator such as actuation stroke and output force will be different from that of the unidirectional configuration (Fig. 2(b)).

As a bending DEA, the actuator configuration presented here (use of a pre-stretched DEA and a silicone substrate) is similar to the dielectric elastomer minimum energy structures (DEMESs) that use a flexible substrate with a hole [22]. DEMESs are also able to achieve bidirectional movement [27]. However, our configuration has several features that dif- 


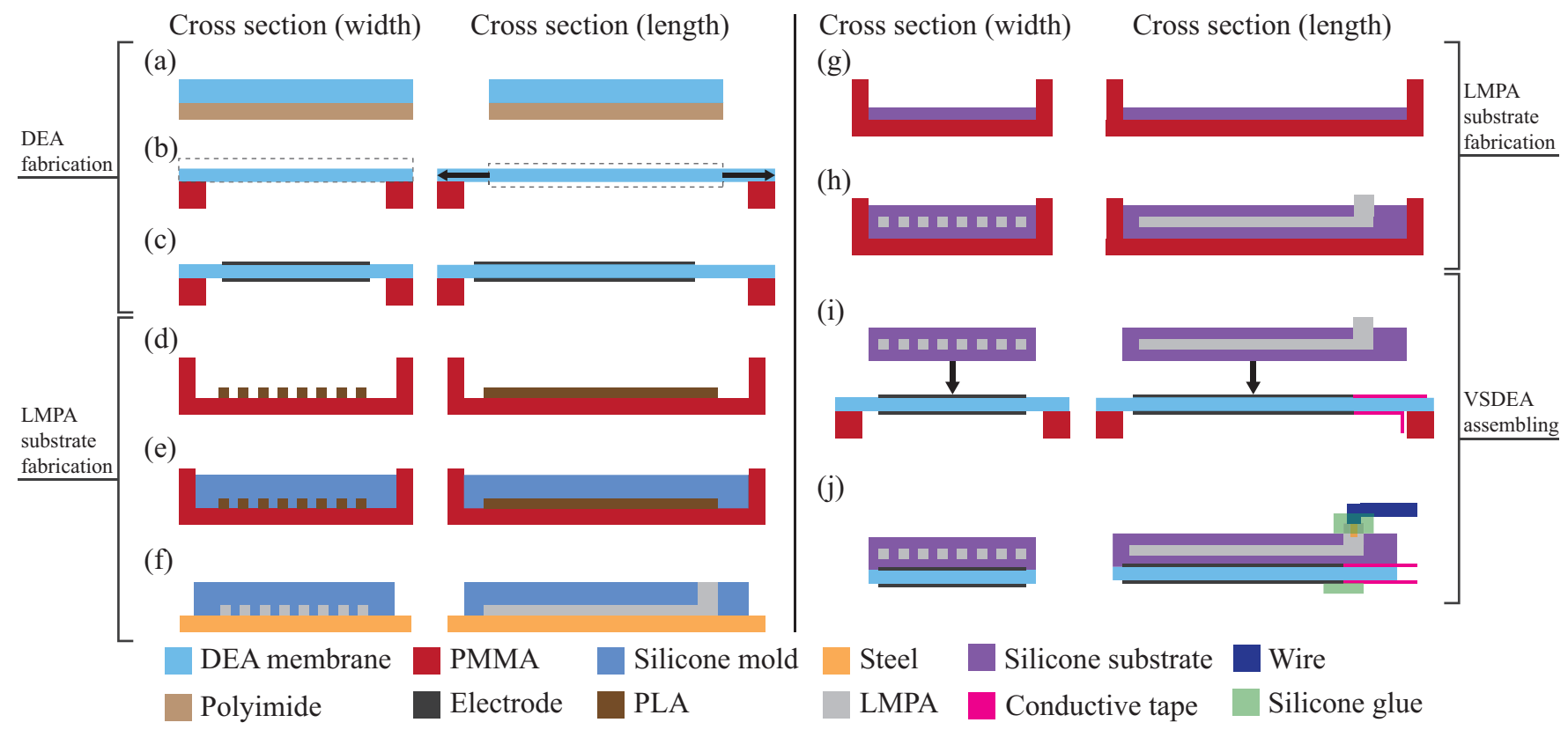

Fig. 3. Fabrication process of VSDEA. (a) The DEA elastomer is cast and (b) pre-stretched followed by (c) the patterning of the electrodes. (d) PLA is printed onto a PMMA mold to form the geometry of the LMPA channel. (e) Fill the mold with Ecoflex. (f) Hot liquid LMPA was filled inside the Ecoflex negative mold. (g) A soft silicone is put in a PMMA mold to encapsulate the LMPA. (h) Place the LMPA and fill the same silicone. (i) After the curing, bond the LMPA substrate to the DEA. (j) Place wires and then silicone glue over the connections.

fer from DEMESs. (1) The high compliance of the structure provides better physical adaptability to environments (e.g., conformation of gripper fingers to the object being held). (2) The absence of a hole eases the fabrication and the designing (e.g., layout of the LMPA channel), and improves the robustness as the DEA is covered by the substrate. (3) The use of silicone allows encapsulation of the LMPA into the substrate. (4) Bidirectional movement can be achieved with simpler structural configuration, just adding another DEA, contrary to DEMESs that require additional parts [27].

\section{B. Fabrication Process}

We fabricated the actuators used for the characterization according to the fabrication process shown in Fig. 3(a)-(j). The DEA and the LMPA substrate are made separately at first, and then they are bonded together at the end of the process. For the DEA, we used a silicone elastomer (CF192186, NuSil Technology). (a) The elastomer was cast on a polyimide sheet using a film coater (ZAA2300, Zehntner) and a film applicator (ZUA2000, Zehntner) forming a membrane with thickness of $\sim 45 \mu \mathrm{m}$ after the curing at temperature of $80 \mathrm{C}^{\circ}$. (b) The membrane was then pealed-off from the sheet, stretched uniaxially, and held in an acrylic (PMMA) frame. (c) Subsequently, electrodes composed of a soft silicone and carbon black were applied on the both sides using a pad-printing machine (TPM-101, Teca-Print).

For the LMPA substrate, (d) Polylactide (PLA) forming the geometry of the LMPA channel was printed on the bottom plate of a PMMA mold using a 3D printer (Replicator 2, MakerBot). (e) The mold was then filled with a silicone (Ecoflex 0030, Smooth-On). (f) After the curing, the Ecoflex negative mold was placed on a stainless steel plate and the hot liquid LMPA was filled inside using a syringe through a punched hole. The LMPA we used has a melting temperature of $47 \mathrm{C}^{\circ}$ (Cerrolow 117, HiTech Alloys). Once cooled, the molded LMPA was carefully separated from the Ecoflex negative mold. Then, the LMPA was annealed above the melting temperature to achieve a more favorable low-energy shape, as opposed to the forced shape of the mold. This step minimizes separation of the alloy in the final device due to poor wetting to the silicone encapsulation. During this step, the temperature of the steel plate was controlled using a hot plate. (g) To encapsulate the LMPA, a soft silicone (Silbione LSR 4305, Bluestar) was put in a PMMA mold. The amount of the silicone determines the position of the LMPA in the substrate in the thickness direction. (h) Before the filled silicone was cured completely, the molded LMPA was placed on the top, and the same silicone was filled again. The adhesion of the non-fully cured silicone prevents the movement of the LMPA until the entire structure is cured.

(i) After the curing of the silicone at room temperature, the LMPA substrate was carefully separated from the mold, and bonded to the DEA prepared at (c), using oxygen plasma surface activation (Zepto plasma system, diener). The DEA was equipped with the electrical connections using a conductive tape and a conductive silver epoxy. (j) The entire structure was cut from the PMMA frame and the electrical connections for the LMPA were formed using electrical wire. After that, a silicone glue (Sylgard 734, Dow Corning) was put on the electrical connections to ensure their connection and to prevent the leakage of the LMPA when it is in the liquid phase.

Fig. 4(a) shows the fabricated VSDEA. The device has a dimension of $40 \mathrm{~mm}$ length $\times 10 \mathrm{~mm}$ width $\times 1 \mathrm{~mm}$ thickness with mass of $\sim 1 \mathrm{~g}$, where the LMPA forms one continuous channel with equally-spaced lines of width 
$0.45 \mathrm{~mm}$ and thickness of $0.3 \mathrm{~mm}$, covering an area of $35 \mathrm{~mm} \times 8 \mathrm{~mm}$. The DEA has active area of $29 \mathrm{~mm} \times 8 \mathrm{~mm}$. VSDEA is flat after the fabrication, and the activation of the LMPA substrate leads to a bending shape due to the prestretch of the DEA. When compared to an actuator without the LMPA, the rigidity is visible where a nut of mass $2 \mathrm{~g}$ is supported (Fig. 4(b)). On the other hand, the device can be compliant when the LMPA substrate is activated (Fig. 4(c)).

For the fabricated actuator, the heating time necessary to melt the LMPA is $\sim 30 \mathrm{~s}$ at a power input of $\sim 1 \mathrm{~W}$. The heating time can be shorten by increasing the input power. On the other hand, even the time to cool back the LMPA below melting takes $\sim 30 \mathrm{~s}$, more than $\sim 60 \mathrm{~s}$ is necessary to cool the actuator back to room temperature. This is due to the fact that the cooling depends on convection between the surface of the LMPA substrate and the surrounding air [26].

\section{Characterization}

1) Experimental setup: We measured the actuation stroke angle, the blocked force, and the reaction force against a forced displacement of the actuators. Fig. 5(a) inset shows a bending actuation of the VSDEA in the soft state. The actuation stroke angle we took is defined as the tip angle difference from the initial shape i.e., no applied voltage. The actuator was placed in the direction minimizing gravity effects. A CMOS camera and image processing with MATLAB were used to obtain the angle value. The blocked force and the reaction force were taken by putting the probe of a load cell (UF1, Applied Measurement Limited) to the actuator tip perpendicularly at the initial shape (Fig. 5(b) inset). During the characterization, the power input for activating the LMPA substrate was kept at $\sim 0.5 \mathrm{~W}$, while the applied voltage for the DEA was varied from 0 to $3 \mathrm{kV}$.

2) Results: Fig. 5(a) shows the measured actuation stroke angle as a function of the DEA applied voltage for the soft and the rigid states. 6 samples were measured and the average was taken. In the soft state, the actuator showed voltage-controllable actuation with the maximum angle range of $23.7^{\circ}$ at $3 \mathrm{kV}$. On the other hand, the rigid state exhibited almost same shape over the DEA applied voltage, only $0.6^{\circ}$ angle difference was observed at $3 \mathrm{kV}$, representing the high bending stiffness of the structure. Fig. 5(b) shows the measured blocked force as a function of the DEA applied voltage for the rigid and the soft state. Same as the angle, 6 samples were measured and the average was taken. Both the states showed similar maximum force at $3 \mathrm{kV}(2.4 \mathrm{mN}$ for the soft state, and $2.1 \mathrm{mN}$ for the rigid state). The larger deviation in the rigid state may have resulted from the alignment errors of the load cell probe and the small actuation angle of the actuator preventing accurate measurement.

Fig. 5(c,d) show the actuation stroke angle and the blocked force as functions of the DEA applied voltage for one actuator with 5 cycle measurements. The actuator exhibited good repeatability, only a small hysteresis was observed, and this is due to low viscoelasticity of the silicones used for the actuator.

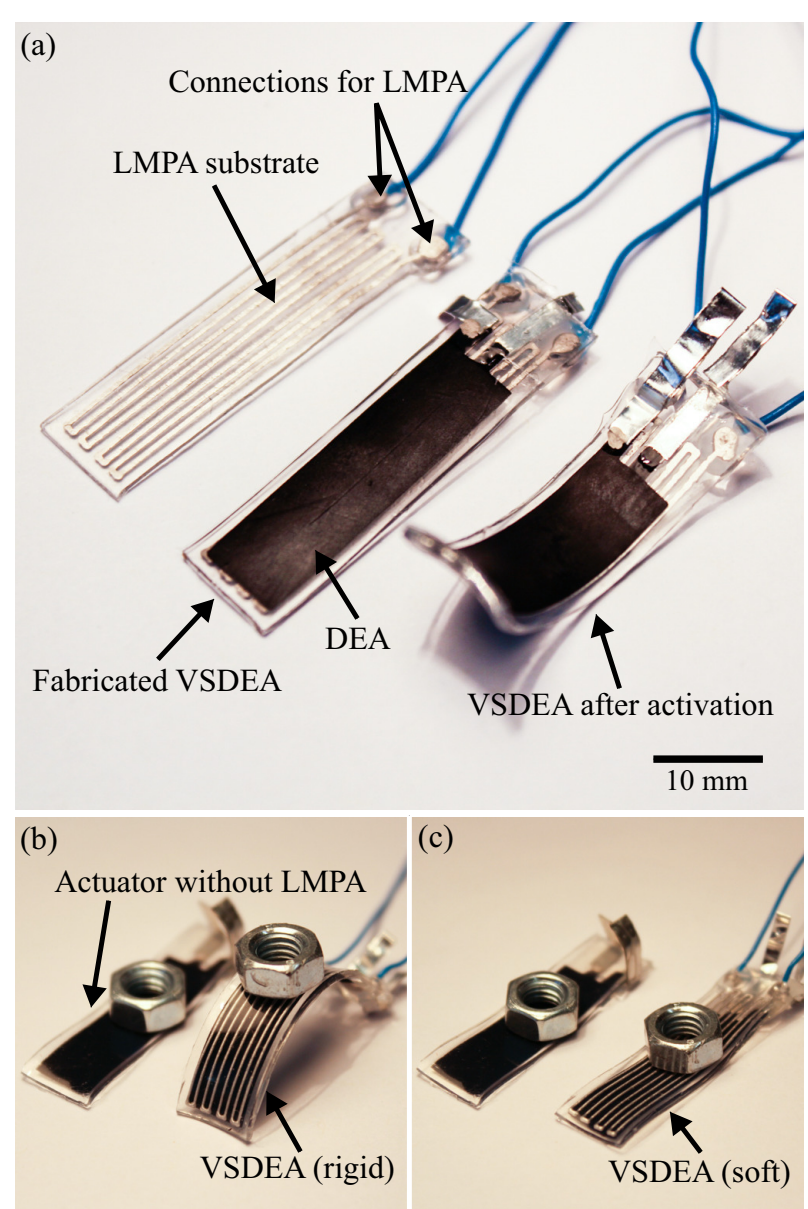

Fig. 4. Fabricated VSDEA. The actuation has an overall dimension of $40 \mathrm{~mm}$ length $\times 10 \mathrm{~mm}$ width $\times 1 \mathrm{~mm}$ thickness. (a) The activation of the LMPA substrate results in a bending shape. (b) Compared to an actuator without the LMPA, the rigidity of VSDEA is visible. (c) The compliance of the device is clear when the LMPA substrate is activated.

Fig. 5(e) shows the measured reaction force as a function of the forced displacement of the load cell probe externally moved by a motorized stage from the initial shape of the actuator. In this measurement, a VSDEA and an actuator without LMPA were used. To clarify their rigidity difference, we estimated the spring constant of the devices $k$ using a least squares method applied to the data on the range of 1-3 mm (linear part). The spring constant $k$ is defined as $k=F_{\mathrm{r}} / x_{\mathrm{d}}$, where $F_{\mathrm{r}}$ and $x_{\mathrm{d}}$ are the reaction force and the forced displacement, respectively. The fitted lines based on the calculation are shown in Fig. 5(e). The spring constant of the VSDEA was obtained as $23.3 \mathrm{mN} / \mathrm{mm}$, and was $\sim 90$ times larger than that of the actuator without the LMPA $(0.26 \mathrm{mN} / \mathrm{mm})$.

\section{Two-finger gripper}

As an application of VSDEA, we developed a two-finger gripper shown in Fig. 1 to demonstrate the usefulness of the actuator. The gripper consists of two VSDEAs connected via plastic parts to act as fingers. Each finger is the same actuator characterized in the previous subsection, therefore, the mass of the active parts of the gripper is $\sim 2 \mathrm{~g}$. The ability of the gripper was demonstrated by picking up an 


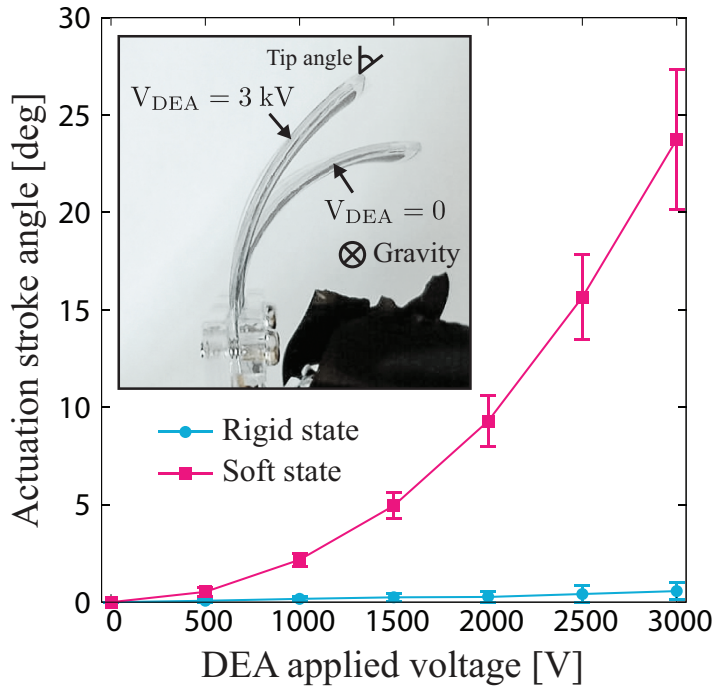

(a)

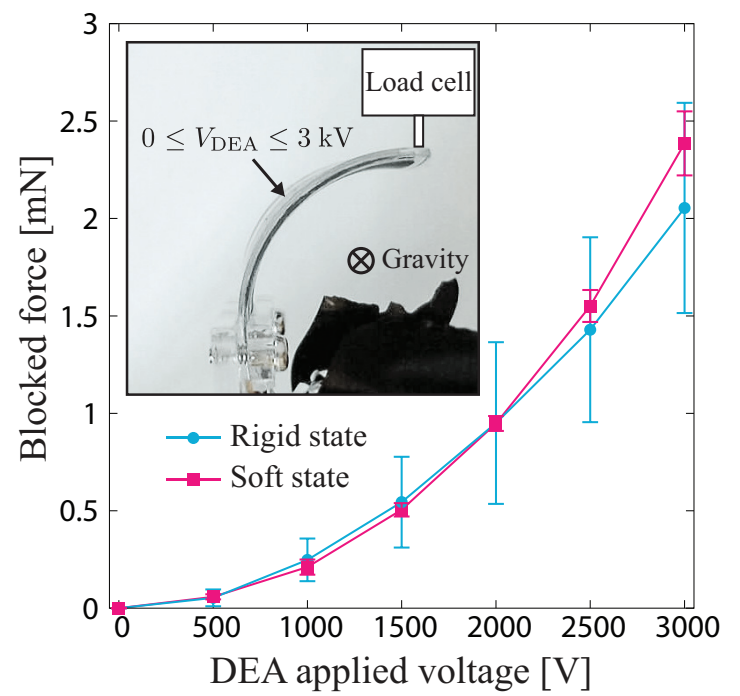

(b)

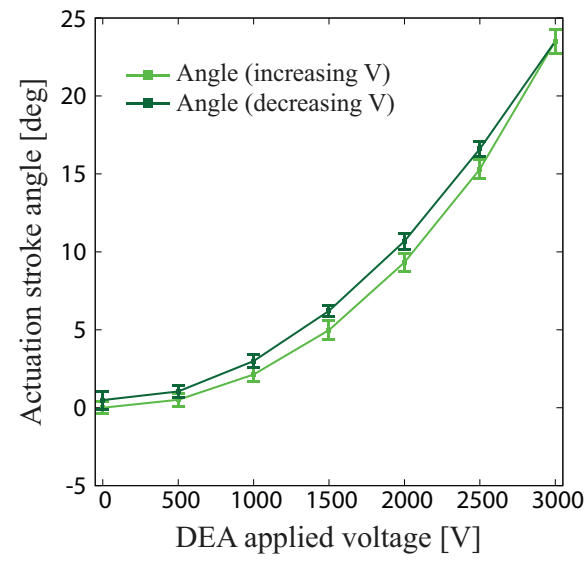

(c)

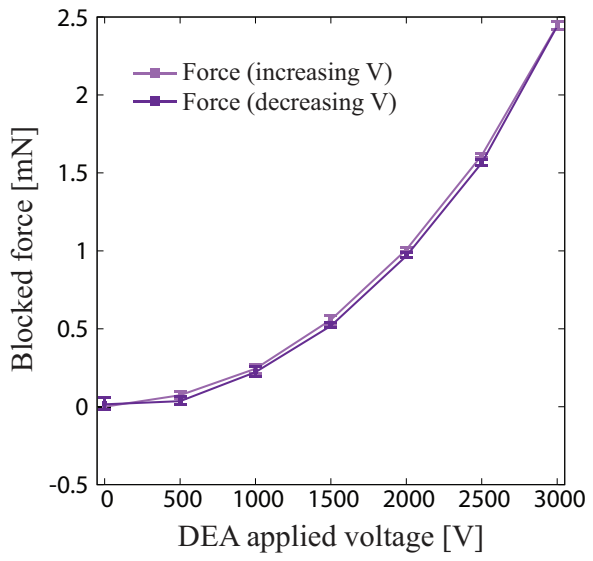

(d)

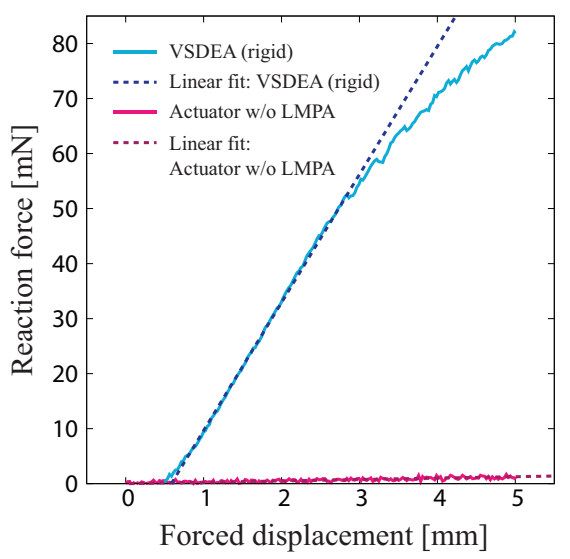

(e)

Fig. 5. (a) Measured actuation stroke angle as a function of the DEA applied voltage. Inset shows actuation of a VSDEA in the soft state. The actuation stroke angle was measured as the tip angle difference from the initial shape. In the soft state, the power input for the LMPA was kept $\sim 0.5 \mathrm{~W}$. The maximum actuation range was observed as $23.7^{\circ}$ for the soft state, and $0.6^{\circ}$ for the rigid state at $3 \mathrm{kV}$. (b) Measured blocked force as a function of the DEA applied voltage. The blocked force was measured by putting the load cell probe on the tip of the actuator. Similar maximum force was observed for both states: $2.4 \mathrm{mN}$ for the soft state, and $2.1 \mathrm{mN}$ for the rigid state. For the stroke angle and the force, the averages and standard deviations are from measurements of 6 samples. (c) Result of 5 cycle measurements of one sample on the actuation stroke angle and (d) the blocked force. The actuator showed good repeatability. (e) The reaction force as a function of the forced displacement. The rigidity of the VSDEA led to a clear difference compared to an actuator without the LMPA. The dashed lines represent the spring constant of the devices obtained by a least squares method.

object, a plastic dish filled with metal washers mass of $11 \mathrm{~g}$. A linear motorized stage was used to provide up and down motion of the device. In the soft state, the gripper wraps the fingers around the object by the actuation of the DEA. After solidifying the fingers, the gripper successfully picked up the object thanks to the high rigidity. The full procedure of the gripper demonstration described above can be seen on [28].

\section{DISCUSSION AND CONCLUSIONS}

A novel variable stiffness actuator VSDEA has been developed. The actuator performs voltage-controlled bending actuation with large deflection $\left(23.7^{\circ}\right)$ and good repeatability in the soft state. In the rigid state, it can maintain a fixed shape, and it has a much larger spring constant $(\sim 90 \times)$ compared to an actuator without LMPA. The fact that both the actuation and the controllable stiffness are generated from a simple composite is a key novelty of VSDEA. The simple structure leads to a high design flexibility of the actuator. The gripper we developed shown in Fig.1 has demonstrated a practical use of VSDEA illustrating its high performance.

There are other potential applications where the actuator could be applied. Since the device does not require any power input to sustain the rigid state, this feature may contribute to the perching of mobile robots, e.g., a flying robot where the available power source is limited [29]. The controllable stiffness allows us to have variable mechanical impedance which would enable various dynamic behaviors of the system e.g., asymmetric deformation of a periodically actuated structure could produce one way thrust like a jellyfish [30]. The current structure of VSDEA does not limit segmentation of the DEA and the LMPA. This means that even from a single device, multi-degree-of-freedom actuation could be generated. Especially the segmentation of the LMPA may 
enable local stiffening of the robot, which could be used for dextrous manipulators (e.g., [8], [31]) and endoscopes (e.g., [32]). The self-sensing capability of both the DEA and the LMPA substrate in VSDEA would support controlling of those applications.

Our future work includes further characterization of VSDEA including the bidirectional configuration such as the detailed stiffness change, the response speed of the actuation, and heating/cooling time. Modeling of the device for establishing design and optimization principles on both the actuation and the variable stiffness characteristics should also be carried out. Also, scalability should be studied to facilitate the creation of larger and smaller structures.

VSDEA is a new technology that will contribute to the development of highly adaptable soft robots thanks to its simplicity and multifunctionality.

\section{ACKNOWLEDGMENTS}

The authors would like to thank O. A. Araromi, S. Mintchev, and T. Akiyama for their helpful comments and discussions. This work was supported by the Swiss National Centre of Competence in Research (NCCR) Robotics, and the Swiss National Science Foundation grant 200020153122 .

\section{REFERENCES}

[1] R. F. Shepherd, F. Ilievski, W. Choi, S. A. Morin, A. A. Stokes, A. D. Mazzeo, X. Chen, M. Wang and G. M. Whitesides, "Multigait soft robot," Proceedings of the National Academy of Sciences, vol. 108, no. 51, pp. 20400-20403, 2011.

[2] F. Ilievski, A. D. Mazzeo, R. F. Shepherd, X. Chen and G. M. Whitesides, "Soft Robotics for Chemists," Angewandte Chemie International Edition, vol. 50, no. 8, pp. 1890-1895, 2011.

[3] H.-T. Lin, G. G. Leisk and B. Trimmer, "GoQBot: a caterpillarinspired soft-bodied rolling robot," Bioinspiration \& Biomimetics, vol. 6, no. 2, pp. 026007-1-026007-14, 2011.

[4] M. T. Tolley, R. F. Shepherd, B. Mosadegh, K. C. Galloway, M. Wehner, M. Karpelson, R. J. Wood and G. M. Whitesides, ”A Resilient, Untethered Soft Robot," Soft Robotics, vol. 1, no. 3, pp. 213-223, 2014.

[5] H. Nakai, Y. Kuniyoshi, M. Inaba and H. Inoue, "Metamorphic robot made of low melting point alloy," in IEEE/RSJ International Conference on Intelligent Robots and System, 2002, pp. 2025-2030.

[6] E. Steltz, A. Mozeika, N. Rodenberg, E. Brown, H. M. Jaeger, ”JSEL: Jamming Skin Enabled Locomotion," in IEEE/RSJ International Conference on Intelligent Robots and Systems, 2009, pp. 5672-5677.

[7] E. Brown, N. Rodenberg, J. Amend, A. Mozeika, E. Steltz, M. R. Zakin, H. Lipson and H. M. Jaeger, "Universal robotic gripper based on the jamming of granular material," Proceedings of the National Academy of Sciences, vol. 107, no. 44, pp. 18809-18814, 2010.

[8] N. G. Cheng, M. B. Lobovsky, S. J. Keating, A. M. Setapen, K. I. Gero, A. E. Hosoi and K. D. Iagnemma, "Design and Analysis of a Robust, Low-cost, Highly Articulated Manipulator Enabled by Jamming of Granular Media," in IEEE International Conference on Robotics and Automation, 2012, pp. 4328-4333.

[9] A. Sadeghi, L. Beccai and B. Mazzolai, "Innovative Soft Robots Based on Electro-Rheological Fluids," in IEEE/RSJ International Conference on Intelligent Robots and Systems, 2012, pp. 4237-4242.

[10] C. Majidi and R. J. Wood, "Tunable elastic stiffness with microconfined magnetorheological domains at low magnetic field," Applied Physics Letters, vol. 97, no. 16, p. 164104, 2010.

[11] Y. Chen, J. Sun, Y. Liu and J. Leng, "Variable stiffness property study on shape memory polymer composite tube," Smart Materials and Structures, vol. 21, no. 9, p. 094021, 2012.

[12] X. Niu, X. Yang, P. Brochu, H. Stoyanov, S. Yun, Z. Yu and Q. Pei. "Bistable Large-Strain Actuation of Interpenetrating Polymer Networks," Advanced Materials, vol. 24, no. 48, pp. 65136519, 2012.
[13] V. Birman, "Review of Mechanics of Shape Memory Alloy Structures," Applied Mechanics Reviews, vol. 50, no. 11, pp. 629-645, 1997.

[14] R. Pelrine, R. Kornbluh, Q. Pei and J. Joseph, "High-speed electrically actuated elastomers with strain greater than 100\%," Science, vol. 287, no. 5454, pp. 836-839, 2000.

[15] I. A. Anderson, T. A. Gisby, T. G. McKay, B. M. O'Brien and E. P. Calius, "Multi-functional dielectric elastomer artificial muscles for soft and smart machines," Journal of Applied Physics, vol. 112 no. 4, p. 041101, 2012.

[16] R. Kornbluh, R. Peirine, Q. Pei, S. Oh and J. Joseph, ”Ultrahigh strain response of field-actuated elastomeric polymers," in SPIE, 2000, vol. 3987, pp. 51-64.

[17] T. A. Gisby, E. P. Calius, S. Xie and I. A. Anderson, "An adaptive control method for dielectric elastomer devices," in SPIE, 2008, vol. 6927, p. 69271C.

[18] T. Li, C. Keplinger, R. Baumgartner, S. Bauer, W. Yang and Z. Suo, "Giant voltage-induced deformation in dielectric elastomers near the verge of snap-through instability," Journal of the Mechanics and Physics of Solids, vol. 61, no. 2, pp. 611-628, 2013.

[19] R. Pelrine, R. Kornbluh, Q Pei, S. Stanford, S Oh, J. Eckerle, R. J. Full, M. A. Rosenthal, K. Meijer, "Dielectric elastomer artificial muscle actuators: toward biomimetic motion," in SPIE, 2002, vol. 4695, pp. 126-137.

[20] C. T. Nguyen, H. Phung, T. D. Nguyen, C. Lee, U. Kim, D. Lee, H. Moon, J. Koo, J.-D. Nam and H. R. Choi, "A small biomimetic quadruped robot driven by multistacked dielectric elastomer actuators," Smart Materials and Structures, vol. 23, no. 6, 2014.

[21] M. T. Petralia and R. J. Wood, "Fabrication and analysis of dielectricelastomer minimum-energy structures for highly-deformable soft robotic systems," in IEEE/RSJ International Conference on Intelligent Robots and Systems, 2010, pp. 2357-2363.

[22] G. Kofod, W. Wirges, M. Paajanen and S. Bauer, "Energy minimization for self-organized structure formation and actuation," Applied Physics Letters, vol. 90, no. 8, p. 081916, 2007.

[23] O. A. Araromi, I. Gavrilovich, J. Shintake, S. Rosset, M. Richard, V. Gass and H. Shea, "Roll-able Multisegment Dielectric Elastomer Minimum Energy Structures for a Deployable Microsatellite Gripper," IEEE/ASME Transactions on Mechatronics, vol. 20, no. 1, pp. 438446, 2015.

[24] C. Jordi, S. Michel and E. Fink, "Fish-like propulsion of an airship with planar membrane dielectric elastomer actuators," Bioinspiration \& Biomimetics, vol. 5, no. 2, p. 026007, 2010.

[25] J. Shintake, S. Rosset, B. Schubert, D. Floreano and H. Shea, "A Foldable Antagonistic Actuator," IEEE/ASME Transactions on Mechatronics, vol. PP, no. 99, pp. 1-12, 2014, doi: 10.1109/TMECH.2014.2359337.

[26] B. Schubert and D. Floreano, "Variable stiffness material based on rigid low-melting-point-alloy-microstructures embedded in soft poly(dimethylsiloxane) (PDMS)," RSC Advances, vol. 3, no. 46, pp. 24671-24679, 2013.

[27] M. Follador, A. T. Conn, B. Mazzolai and J. Rossiter, "Active-elastic bistable minimum energy structures," Applied Physics Letters, vol. 105, no. 14, p. 141903, 2014.

[28] J. Shintake, B. Schubert, S. Rosset, H. Shea and D. Floreano, "Variable Stiffness Actuator for Soft Robotics Using Dielectric Elastomer and Low-Melting-Point Alloy," YouTube, 2015. Available: https://www.youtube.com/watch?v=y1KcaiKpzXU.

[29] C. E. Doyle, J. J. Bird, T. A. Isom, J. C. Kallman, D. F. Bareiss, D. J. Dunlop, R. J. King, J. J. Abbott and M. A. Minor, "An AvianInspired Passive Mechanism for Quadrotor Perching," IEEE/ASME Transactions on Mechatronics, vol. 18, no. 2, pp. 506-517, 2013.

[30] J. C. Nawroth, H. Lee, A. W. Feinberg, C. M. Ripplinger, M. L. McCain, A. Grosberg, J. O. Dabiri and K. K. Parker, ”A tissue-engineered jellyfish with biomimetic propulsion," Nature Biotechnology, vol. 30, no. 8, pp. 792797, 2012.

[31] A. D. Marchese, R. K. Katzschmann and D. Rus, "Whole Arm Planning for a Soft and Highly Compliant 2D Robotic Manipulator," in IEEE/RSJ International Conference on Intelligent Robots and Systems, 2014, pp. 554-560.

[32] A. J. Loeve, O. S. van de Ven, J. G. Vogel, P. Breedveld and J. Dankelman, "Vacuum packed particles as flexible endoscope guides with controllable rigidity," Granular Matter, vol. 12, no. 6, pp 543554 . 